\title{
Development and Evaluation of Laboratory Bioassays to Study Powdery Mildew Pathogens of Phlox In Vitro
}

\author{
Coralie Farinas, ${ }^{1}$ Pablo Jourdan, ${ }^{2}$ Pierce A. Paul, ${ }^{3}$ and Francesca Peduto Hand ${ }^{1, \dagger}$ \\ ${ }^{1}$ Department of Plant Pathology, The Ohio State University, Columbus, OH 43210 \\ ${ }^{2}$ Department of Horticulture and Crop Science, The Ohio State University, Columbus, OH 43210 \\ ${ }^{3}$ Department of Plant Pathology, The Ohio State University, Wooster, OH 44691
}

\begin{abstract}
The genus Phlox consists of approximately 65 species that include some of the most prevalent ornamental plants in the temperate zone. These popular ornamentals are extremely susceptible to powdery mildew (PM) caused by the biotrophic fungi Golovinomyces magnicellulatus and Podosphaera sp. In this study, we used Phlox paniculata and $P$. glaberrima to develop a set of laboratory tools to study these pathogens in vitro, including a detached leaf and a micropropagated plantlet bioassay. We assessed pathogen growth under different experimental conditions, which included the use of four different media variations (1/2 MS medium amended with benzimidazole and tetracycline), three ages of pathogen culture $(14,18$, and 22 days), three phenological stages of the host tissue (1st, 3rd, and 5th node leaves), placement of inoculum

on both leaf surfaces (abaxial and adaxial), and three different inoculation techniques (single spore transfer, colony tapping, colony brushing). Detached $P$. paniculata leaves were successfully maintained on benzimidazole-amended 1/2 MS medium for up to 3 weeks. For both pathogens, the use of 18-day-old cultures resulted in a higher number of larger, higher sporulating colonies compared with 1-4 and 22-dayold cultures. The adaxial side of 3rd node leaves supported statistically significant more fungal growth compared with the adaxial side of 1 st and 5th node leaves. Both pathogens also successfully infected micropropagated plantlets of $P$. glaberrima. These newly developed tools should facilitate in vitro studies on PM of Phlox and possibly be applicable to other ornamental species attacked by the same fungi.
\end{abstract}

Phlox L. is a genus of ornamental plants in the family Polemoniaceae comprised of approximately 65 species that are characterized by colorful, long-lasting flowers displaying mostly monochromatic tones of white, blue, violet, and pink. The genus is native to North America, inhabiting very diverse habitats, from marshes and riverbanks to prairies, deserts, and tundra (Locklear 2011; Zale and Jourdan 2015; Zale et al. 2016). Almost all the species are herbaceous or semiwoody perennials, only a few are annuals. Plant height varies from several centimeters in species with creeping habit to over $1 \mathrm{~m}$ in the upright herbaceous species (Coombs 2017; Locklear 2011; Zale and Jourdan 2015; Zale et al. 2016). The genus plays an important role in horticulture, as it is widely used in gardens and landscapes, both in formal and naturalistic arrangements. Within the three billion dollar horticultural industry in the United States, Phlox accounts for over \$12 million in annual national sales (wholesale and retail combined value; NASS 2014).

A major constraint to the use of Phlox in landscapes, and a constant burden for nursery growers, is its susceptibility to powdery mildew (PM; Armitage 2008; Coombs 2017; Zale and Jourdan 2015). Under conditions of high relative humidity and warm temperatures, the disease drastically limits plant development, and plantings may become unsightly. Affected plants display powdery growth of the fungal colonies on the surface of flowers, leaves, and stems that reduce the

\section{${ }^{\dagger}$ Corresponding author: F. Peduto Hand; hand.81@osu.edu}

Funding: This work was partially funded by the Fred C. Gloeckner Foundation through the 2016 New York Florists' Club Grant, the USDA-NIFA Hatch project \#1004939, and The Ohio State University Department of Plant Pathology.

*The $\boldsymbol{e}$-Xtra logo stands for "electronic extra" and indicates that two supplementary tables are published online.

The authors declare no conflict of interest.

Accepted for publication 11 January 2019.

() 2019 The American Phytopathological Society plant's photosynthetic ability (McGrath and Shishkoff 2001). Infected leaves become chlorotic, then necrotic, and eventually fall off (Hermann 1994). Severe infections can cause extensive leaf drop, leading to plant death (Coombs 2017). Due to the high degree of susceptibility to the disease, Phlox cultivation heavily relies on the use of ecologically and economically expensive fungicides, which are applied as frequently as every 10 to 14 days throughout the growing season to protect the ornamental value of the plant (Armitage 2008).

Powdery mildew pathogens are biotrophic parasitic fungi encompassing different genera and species. These fungi infect susceptible hosts through dormant mycelium in buds or by wind-dispersed conidia, which are continuously produced in chains on the mycelium. In late summer, chasmothecia are formed from which ascospores are released the following season by a special form of dehiscence known as vertical slits (Braun et al. 2002). Different PM fungi are known to infect Phlox in the United States. A recent survey conducted in Ohio showed that Podosphaera sp. and Golovinomyces magnicellulatus (U. Braun) V.P. Heluta are predominant (Farinas and Peduto Hand 2017).

Since PM fungi are biotrophic organisms, studies involving these pathogens need to be conducted on living plants. However, in vitro bioassays have been developed for many PM-crop systems (extensively reviewed by Nicot et al. 2002), including rose (Linde and Debener 2003), rhododendron (Kenyon et al. 1995), barley (SurlanMomirovic et al. 2016), grape (Peduto et al. 2013), and cucurbits (McGrath and Shishkoff 2001) among others. These bioassays have facilitated the study of PM pathogens, their biology and epidemiology, and the susceptibility of host populations to the disease they cause. Thus, the availability of these laboratory tools provides an opportunity to study these biotrophic organisms under controlled conditions.

Most of the published literature on Phlox PM has focused on the screening of different species and cultivars for resistance to the disease (Bir and Conner 2002; Hawke 1999, 2011; Thompson and Svejda 1965). These studies have been carried out exclusively in field trials, typically relying on natural infection of the plant. In vitro bioassays would be helpful tools for carrying out such studies, as they would provide the benefit of controlled experimental conditions (including infection). However, at present, no record of a laboratory 
bioassay for the Phlox-PM system has been found in the literature. Therefore, the objectives of this study were to develop (i) a detached leaf bioassay, (ii) a micropropagated plantlet bioassay, and (iii) to optimize the experimental conditions for their use in studying PM pathogens of Phlox in vitro.

\section{Materials and Methods}

Plant material. The plant materials used in this study included both adult potted plants and micropropagated plantlets. Bare root adult plants of Phlox paniculata cv. Starfire were purchased from a commercial grower in May 2017 and February 2018 and transplanted into 3-gal nursery pots containing Metromix 360 growing medium (Sun Gro Horticulture, Bellevue, WA) amended with perlite at a ratio of 3:1. Eight grams of Osmocote 14-14-14 slow release fertilizer (ICL Specialty Fertilizers, Dublin, OH) were spread on the soil surface of each pot at transplant, and then plants were further fertilized with 150 ppm of Peters Professional 20-10-20 water-soluble fertilizer (ICL Specialty Fertilizers, Dublin, $\mathrm{OH}$ ) as needed. One set of plants was maintained in a greenhouse at a temperature between 20 and $25^{\circ} \mathrm{C}$, and supplemental lighting (Plantmax Metal Halide Sky Blue 600 watt) was used during the winter months to maintain a 14-h photoperiod. Another set of plants was maintained in a growth chamber (Conviron PGR-15) at a temperature of $24^{\circ} \mathrm{C}$ during the day and $22^{\circ} \mathrm{C}$ at night, with a $14-\mathrm{h}$ photoperiod. To prevent natural powdery mildew infection in the greenhouse, plants were sprayed weekly to run-off with a $20 \%$ raw (i.e., unpasteurized) milk solution using a hand-pump sprayer. Every 2 months, plants were cut back to allow new vegetative growth.

Pathogen inoculum. PM-infected Phlox paniculata plants collected from two botanical gardens, one in Ohio and one in Virginia, were used to produce single spore cultures for this study. Infected leaves were pressed against the surface of an empty sterile petri dish to dislodge conidia from the conidiophores. Under a dissecting microscope (Leica S6D), single conidia were picked up from the dish using an ethanol-disinfected eyelash glued to a wooden stick, and transferred on disinfested $P$. paniculata leaves maintained inside $100 \times 15 \mathrm{~mm}$ petri dishes containing $25 \mathrm{ml}$ of half-strength Murashige and Skoog (i.e., 1/2 MS) medium (Caisson Labs) and $2.5 \mathrm{mg} / \mathrm{liter}$ of Gelrite (PlantMedia, bioWorld). Inoculated leaves were incubated on a laboratory shelf under white light (Philips F54T5/841) with a 14-h photoperiod at $24 \pm 1^{\circ} \mathrm{C}$. After 2 weeks, and every 2 to 3 weeks thereafter, each newly developed sporulating colony was transferred onto a new disinfected leaf by cutting the leaf tissue around the colony with a sterile scalpel and then pressing the colony onto the new leaf. Isolate species identification was carried out through rDNA ITS sequence analysis. To this extent, total genomic DNA was extracted from single spore cultures using a Chelex extraction method (Walsh et al. 1991), and a nested PCR was performed using the primer pairs ITS5/P3 and ITS5/ITS4 according to Matsuda and Takamatsu (2003). Isolates of G. magnicellulatus and Podosphaera sp. were used in separate experiments, for all the experiments described below.

Detached leaf bioassay. In order to develop a detached leaf bioassay, leaves on the second and third nodes from the apical meristem were harvested from the potted plants and transferred to the laboratory where they were initially washed with a $0.067 \%$ dish soap solution (Dawn, Procter \& Gamble) and rinsed three times with Milli-Q Type 1 Ultrapure Water (Milli-Q, EMD Millipore). Leaves were then disinfected in a $2 \%$ sodium hypochlorite solution for 5 min and further rinsed three times with sterile ultrapure water. Following disinfection, leaves were blotted dry using sterile paper towels before being placed on the surface of agar media, inside a laminar flow hood.

Effects of different media on the health of detached leaves over time. Four different types of media in $100 \times 15 \mathrm{~mm}$ petri dishes were tested for their ability to maintain the health of detached Phlox leaves in vitro over time. One-half strength MS medium containing $2.5 \mathrm{mg} /$ liter of Gelrite was used as the base medium (medium 1) and was compared with three different variations: one containing $0.01 \%$ of the fungicide benzimidazole (medium 2); one containing $0.01 \%$ of the antibiotic tetracycline (medium 3); and one containing the combination of both (medium 4). Each treatment was tested on six replicated plates, which were placed in stacks of four and arranged in a completely randomized design on a laboratory shelf under white light with a 14-h photoperiod at $24 \pm 1{ }^{\circ} \mathrm{C}$. Every day, plates in each stack were rotated, in order to be exposed to the same daily amount of light. The severity of leaf chlorosis and necrosis (i.e., leaf damage), expressed as percentage of leaf area covered by the symptoms, was visually assessed on each leaf every 4 days for 3 weeks, after which the area under the leaf damage progress curve (AULDPC) was calculated (Madden et al. 2007). The experiment was conducted twice.

Development of powdery mildew on detached leaves. To define the optimum conditions for PM growth on a detached leaf bioassay, three factors were considered: age of the culture, phenology of the host tissue, and side of the leaf surface where inoculum is placed. The first factor was tested in one experiment, and the second two factors were tested in combination in a second experiment, both carried out using medium 2 described above. Each experiment was conducted at least twice, and each was composed of six replicated plates arranged in a completely randomized design and incubated on a laboratory shelf as previously described for 15 days.

The effect of age of pathogen culture was tested by comparing spores transferred from 22-, 18-, and 14-day-old PM colonies onto the test leaves. The effect of the phenology of the host tissue was tested in a separate experiment by comparing the use of young leaves with obvious anthocyanin presence (i.e., 1st node leaves), middleaged leaves transitioning to green color (i.e., 3rd node leaves), and older leaves completely green in color (i.e., 5th node leaves; Fig. 1). In addition, the effect of leaf surface was tested by comparing the placement of inoculum on the abaxial or adaxial side of the leaf. The two treatment factors (i.e., host phenology and leaf surface) were arranged in a complete factorial experiment consisting of one plate for each factor combination, replicated 6 times.

In all experiments, the pathogen was inoculated by excising a sporulating colony from an infected leaf and pressing the colony onto eight places on the surface of a disinfected healthy leaf (Fig. 2).

Data collection. Treatment effect on PM development was assessed by calculating the incidence of PM infection, and by measuring the size of the developing colony and the spore production per unit of colony area over time. Because colonies were not circular, colony size was measured by recording the maximum length and width of each colony. To do so, colonies were observed under a dissecting microscope (Leica DM750), and pictures of each colony were taken with a microscope camera (Leica EC4) to subsequently record the aforementioned measurements using the software LAS EZ (Leica Microsystems). The two measurements of each colony were averaged to estimate the size of the colony. Treatment effect on spore production of each growing colony was assessed by excising 4-mm leaf disks from each sporulating colony on each leaf using a cork borer and by placing them in individual microcentrifuge tubes containing $0.5 \mathrm{ml}$ of a $0.1 \%$ Tween- 20 water solution. Tubes were vortexed for $20 \mathrm{~s}$, then centrifuged for $2 \mathrm{~min}$ at 15,000 rpm, then vortex again for $5 \mathrm{~s}$ before spore counts. Spores were counted using a hemacytometer, and two counts were averaged. Spore density was derived by dividing counts by colony area according to the following formula:

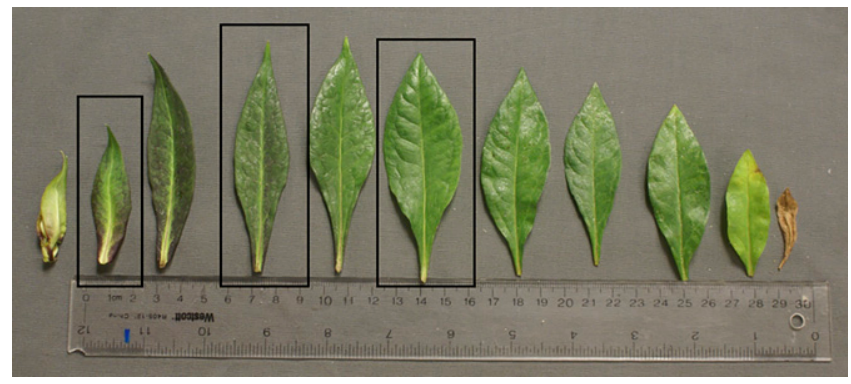

Fig. 1. Example of different phenological stages of Phlox paniculata cv. Starfire leaves Highlighted with rectangular boxes (from left to right) are 1st, 3rd, and 5th node leaves. 
average spore number $\times$ hemacytometer constant $\times$ volume of spore suspension/leaf disk area.

Micropropagated plantlet bioassay. Plantlets of $P$. glaberrima were micropropagated from a collection maintained at the Ornamental Plant Germplasm Center (OPGC) in Columbus, OH. Stem cuttings were transferred to tubes containing $15 \mathrm{ml}$ of MS medium supplemented with $8.88 \times 10^{-06} \mathrm{M}$ of 6-Benzyl amino purine (BAP; Sigma-Aldrich) and $4.75 \mathrm{~g} /$ liter of TC gel (Caisson Labs) to promote shoot production. The plantlets were grown inside an incubator (I-66VL Percival Scientific) under $14 \mathrm{~h}$ light at $25^{\circ} \mathrm{C}$ for 2 months, then transferred to MS medium without cytokinin supplement to promote root growth and incubated under the same conditions as described above for three additional months.

Five-month-old micropropagated plantlets were inoculated with 18-day-old cultures of Podosphaera sp. and G. magnicellulatus in separate experiments. Plantlets were inoculated with an ethanoldisinfected artist fan paintbrush, which was previously thinned to reduce bristle density. Plantlets were removed from their tubes, and a 9-mm colony from an 18-day-old PM culture was brushed on the entire plantlet surface. One PM colony was used per plantlet. Then plantlets were returned to the corresponding tubes. Each experiment had 6 replications and was conducted twice. After inoculation, the plantlets were completely randomized on a laboratory shelf under 14-h photoperiod at $24 \pm 1{ }^{\circ} \mathrm{C}$. Incidence of PM infection expressed as presence or absence of any PM growth on the entire plantlet, and disease severity expressed as percentage of plantlet area covered by PM growth, were visually assessed weekly for 3 weeks postinoculation.

Data analysis. All statistical analyses were conducted using the $\mathrm{R}$ package agricolae v. 1.2-8 (De Mendiburu 2017) and nlme v. 3.1-131 (Pinheiro et al. 2017) in RStudio v. 1.1.383. In all experiments, treatment effects on the AULDPC of severity of leaf damage, disease incidence, disease severity, size of colony, and spore production per unit of area were analyzed by ANOVA, and Tukey HSD test was used to separate means $(\alpha \leq 0.05)$. All spore production data, except for the experiment testing the age of culture using $G$. magnicellulatus, were $\log$ transformed to provide better homogeneity of variance prior to ANOVA analysis, and then back-transformed to present results. All incidence data were arcsine transformed to provide better homogeneity of variance

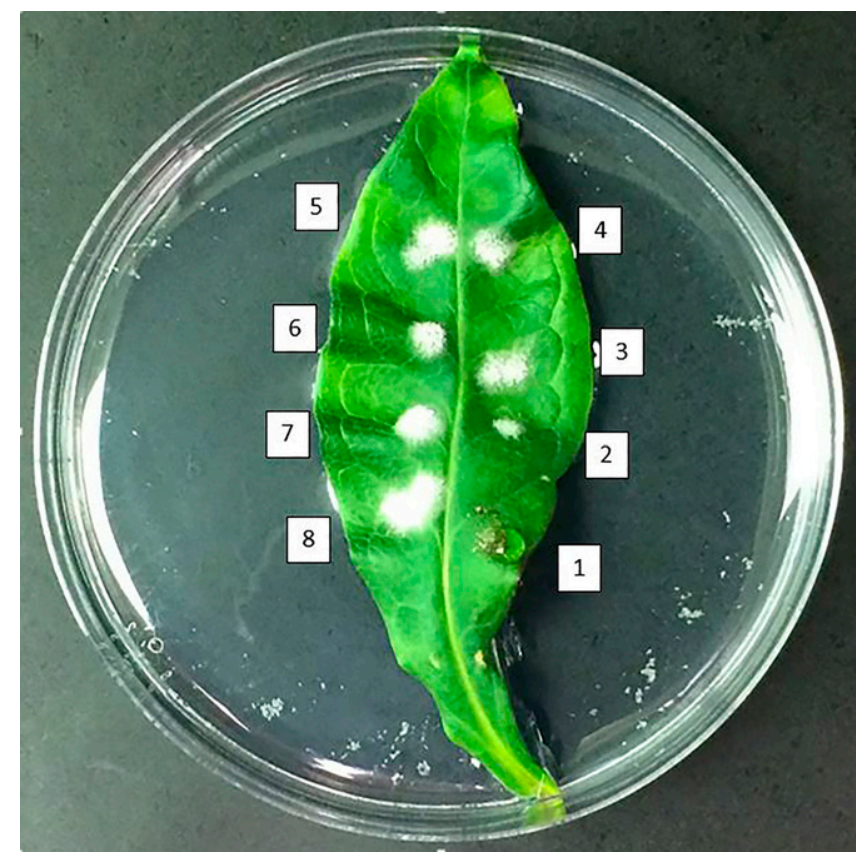

Fig. 2. Example of a detached Phlox paniculata cv. Starfire leaf maintained on $1 / 2 \mathrm{MS}+$ Benzimidazole medium (medium 2). Numbers 1-8 represent the eight subsequent places on the leaf on which a single colony was pressed and from which eight new colonies developed. prior to ANOVA analysis. The different runs of the experiments were treated as random effects and the treatments as fixed effects. Each experimental run was analyzed separately first, then the data were pooled together. Finally, a $\chi^{2}$ test of independence was used to assess if the sequence of inoculation presses on each leaf (1-8; Fig. 2) was related to a chance of a colony developing.

\section{Results}

Detached leaf bioassay. For clarity of reporting, results are presented by each individual factor that was tested in the development of the bioassay.

Effects of different media on the health of detached leaves over time. There was a significant effect of media type on the health of detached leaves over time in the first experimental run but not in the second run (Table 1). For clarity of reporting, data from the two experimental runs were pooled together. Medium 1 showed statistically lower leaf damage than Medium 3 and 4, on which the highest AULDPC was recorded (Fig. 3). Medium 1 and 2 were not significantly different from each other.

Effect of age of culture on the development of PM. For G. magnicellulatus, the $\chi^{2}$ test of independence showed a nonsignificant chance of colonies developing from each inoculation press $\left[\chi^{2}(7\right.$, $n=576)=13.576 ; P>0.05]$. There was a significant effect of age of culture on PM incidence and colony size in the first run of the experiment but not in the second run (Supplementary Table S1). No age of culture effect was observed on spore production and on colony size.

Table 1. Summary statistics of the effect of type of medium on the percentage of leaf damage (chlorosis + necrosis) observed on detached Phlox paniculata leaves over time

\begin{tabular}{|c|c|c|c|}
\hline \multirow[b]{2}{*}{ Factor tested } & \multirow[b]{2}{*}{ Experimental run } & \multicolumn{2}{|c|}{ AULDPC ${ }^{y}$} \\
\hline & & $\boldsymbol{F}$ & $P$ value \\
\hline \multirow[t]{2}{*}{ Type of medium ${ }^{\mathrm{z}}$} & 1 & 5.249 & 0.003 \\
\hline & 2 & 1.2 & 0.321 \\
\hline
\end{tabular}

${ }^{y}$ Area under the leaf damage progress curve.

${ }^{\mathrm{z}}$ Four different media were tested: 1/2 MS; $1 / 2 \mathrm{MS}+$ Benzimidazole; $1 / 2 \mathrm{MS}+$ Tetracycline; 1/2 MS + Benzimidazole + Tetracycline.

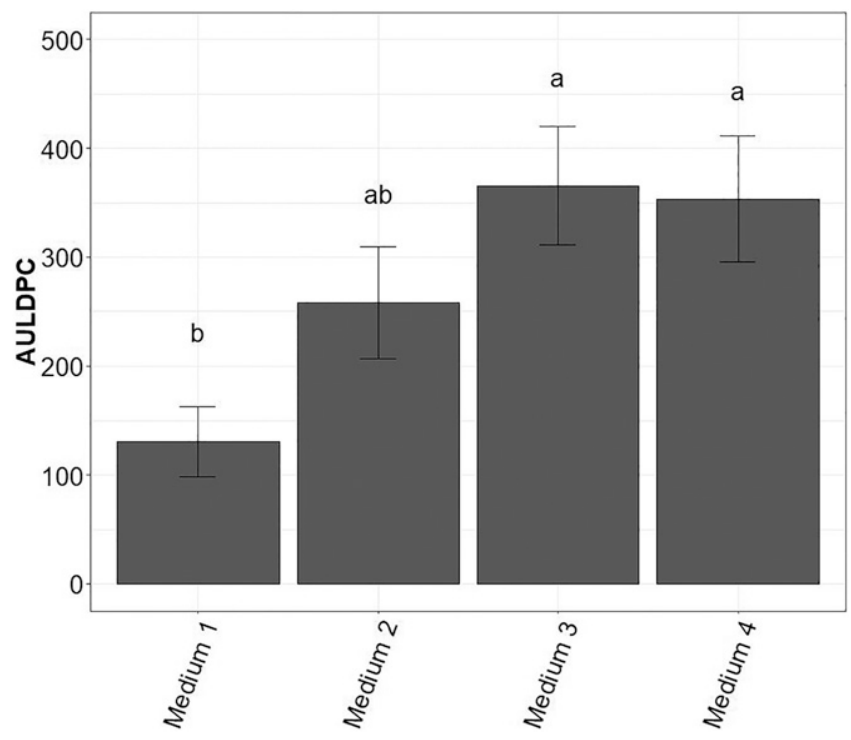

Fig. 3. Area under the leaf damage progress curve (AULDPC) of the percentage of leaf damage (chlorosis + necrosis) recorded every 4 days for 20 days after plating on different media types $(P=0.042)$. Medium 1: 1/2 Murashige and Skoog (MS); Medium 2: 1/2 MS + Benzimidazole; Medium 3: 1/2 MS + Tetracycline; Medium 4: $1 / 2 \mathrm{MS}+$ Benzimidazole + Tetracycline. Columns represent the mean value of two runs of the experiment. Bars indicate \pm SE. Columns followed by the same letter are not statistically different according to Tukey's HSD test $(\alpha=0.05)$. 
Data from the two experimental runs were combined for clarity of reporting. The incidence of PM infection did not differ significantly among the different ages of culture tested (Fig. 4; $P=0.07$ ). High incidence values were recorded in all treatments (range 76 to $90 \%$; Fig. 4). When using 18-day-old cultures as inoculum, the colony size was significantly bigger than when using 22-day-old cultures, but not significantly different than when using 14-day-old cultures. The number of spores produced per unit area was not significantly different among treatments (Table 2).

For Podosphaera sp., the $\chi^{2}$ test of independence also showed a nonsignificant chance of colonies developing from each inoculation press $\left[\chi^{2}(7, n=576)=8.7254 ; P>0.05\right]$. There was a significant effect of age of culture on PM incidence in both experimental runs and on spore production in the first run, but there was no statistically significant effect on colony size in either experimental run.

Data from the two experimental runs were combined for clarity of reporting. Incidence of PM infection was significantly higher on leaves inoculated with 18- compared with 14- and 22-day-old cultures (Fig. $4 ; P \leq 0.001$ ). The colony size was not significantly different among treatments. When using 18-day-old cultures as inoculum, the number of spores produced per colony area was significantly

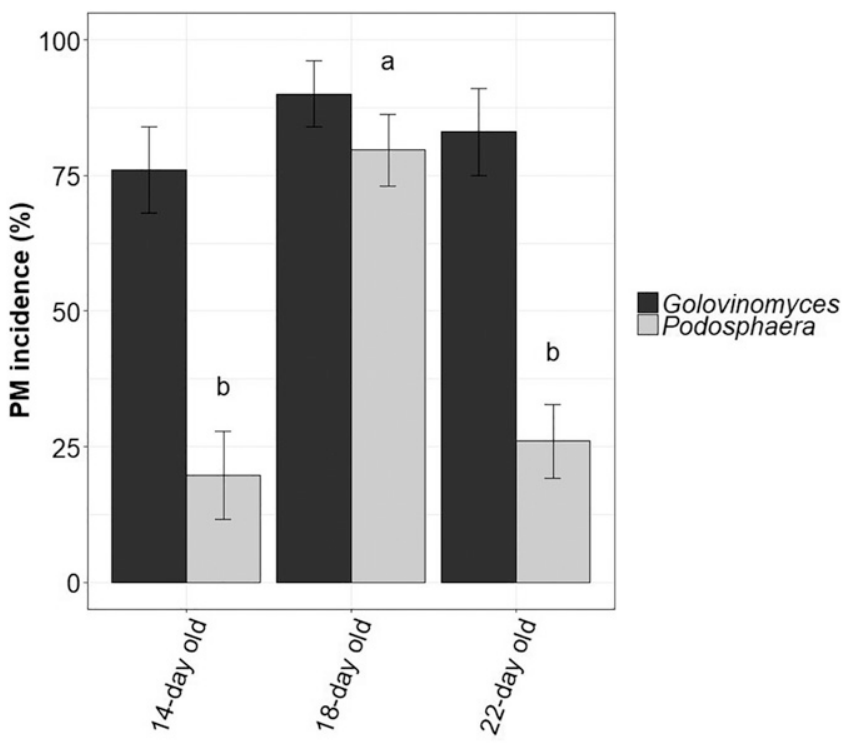

Fig. 4. Percentage of powdery mildew (PM) incidence developed on 3rd node leaves of Phlox paniculata by using 14-, 18-, and 22-day-old cultures of Golovinomyces magnicellulatus $(P=0.07)$ and Podosphaera sp. $(P=<0.001)$. Columns represent the mean value of two runs of the experiment. Bars indicate $\pm \mathrm{SE}$. Columns followed by the same letter are not statistically different according to Tukey's HSD test $(\alpha=0.05)$. Statistical analysis performed on arcsine-transformed data. Untransformed data are displayed. higher than when using 22-day-old cultures, but not significantly different than when using 14-day-old cultures (Table 2).

Effect of phenology of the host tissue and leaf surface on the development of PM. For G. magnicellulatus, there was a significant effect of host phenology (i.e., leaf node) on PM incidence in both experimental runs (Supplementary Table S2). However, the effect of the side of the leaf surface was only significant in the second run. When looking at the colony size, only the side of the leaf surface had a significant effect in the second run. When looking at the spore production per unit of area, the side of the leaf surface had a statistically significant effect in both runs, while the leaf node had statistically significant effect in the second run only. No significant treatment interactions were observed across datasets and experimental runs.

The data were pooled together for clarity of results. Disease incidence on the adaxial side of 3rd node leaves was statistically higher than on 1st and 5th node leaves and was not statistically different than that observed on the abaxial side of all three node leaves (Fig. 5). Disease incidence on the abaxial side of the leaf was not statistically different across leaf nodes (Fig. 5). Within 1st node leaves, the abaxial side had statistically higher incidence than its adaxial side (Fig. 5).

Concerning the size of the colonies, within either the abaxial or adaxial sides, the three node leaves were not statistically different from each other (Table 3). However, within leaf nodes, 1st node leaves showed statistical differences between leaf surfaces, with the abaxial having larger colonies than the adaxial side (Table 3). Third and 5th node leaves did not show statistical differences within side of the leaf surface (Table 3 ).

Concerning spore production, the abaxial sides of the three different node leaves were not statistically different (Table 3). However, colonies growing on the adaxial side of $3 \mathrm{rd}$ node leaves produced significantly more spores than on 1st node leaves (Table 3). Lastly, on 3 rd node leaves, more spores were produced on the abaxial side compared with the adaxial side (Table 3 ).

For Podosphaera sp., there was a significant effect of host phenology (i.e., leaf node) when looking at incidence, colony size, and spore production in the three experimental runs (except the first run of the spore production data $[P=0.06])$. There was not a significant effect of side of the leaf surface when looking at incidence. There was a significant effect of side of the leaf surface in the first run of the colony size data, and in the three experimental runs of the spore production data. No significant treatment interaction was observed when looking at incidence except in the first run.

The results from three experimental runs were combined for clarity of reporting. When looking at disease incidence on the adaxial side of the leaf, 3rd node leaves had statistically higher incidence than 5 th node leaves (Fig. 5). Concerning the abaxial side, no treatment was statistically different (Fig. 5).

Concerning the size of the colony, 3rd node leaves had statistically larger colonies than 5 th node leaves when grown on the adaxial side; however, no statistical difference was observed when growing on the abaxial side (Table 3). No statistical difference was observed between leaf surfaces within each leaf node (Table 3).

Table 2. Effect of the age of pathogen culture on the size of the fungal colonies and the number of spores per unit of area produced by Golovinomyces magnicellulatus and Podosphaera sp. on detached Phlox paniculata leaves

\begin{tabular}{|c|c|c|c|c|c|c|}
\hline \multirow{3}{*}{$\begin{array}{l}\text { Pathogen } \\
\text { Golovinomyces magnicellulatus }\end{array}$} & \multirow{2}{*}{\multicolumn{2}{|c|}{ Parameter }} & \multicolumn{3}{|c|}{ Age of pathogen culture (days) } & \multirow{3}{*}{$\frac{P \text { value }}{0.008}$} \\
\hline & & & \multirow{3}{*}{$\begin{array}{l}\mathbf{1 4} \\
5.45 \mathrm{ab} \\
0.63\end{array}$} & \multirow{3}{*}{$\begin{array}{l}\mathbf{1 8} \\
6.69 \mathrm{a} \\
0.67\end{array}$} & \multirow{3}{*}{$\begin{array}{l}22 \\
4.79 \mathrm{~b} \\
0.47\end{array}$} & \\
\hline & Colony size $(\mathrm{mm})$ & Mean & & & & \\
\hline & & $\mathrm{SE}^{\mathrm{x}}$ & & & & \\
\hline & Spore $/ \mathrm{mm}^{2}$ & Mean & 837 & 1179 & 782 & 0.086 \\
\hline & & SE & 164 & 228 & 165 & \\
\hline Podosphaera sp. & Colony size $(\mathrm{mm})$ & Mean & 2.87 & 3.25 & 2.3 & 0.06 \\
\hline & & $\mathrm{SE}^{\mathrm{x}}$ & \pm 0.46 & \pm 0.37 & \pm 0.28 & \\
\hline & Spore $/ \mathrm{mm}^{2}$ & Meany & $83.1 \mathrm{ab}$ & $81.5 \mathrm{a}$ & $13.5 \mathrm{~b}$ & 0.021 \\
\hline & & $95 \% \mathrm{CL}^{\mathrm{z}}$ & 0,213 & 0,142 & 5,53 & \\
\hline
\end{tabular}

${ }^{x} \mathrm{SE}=$ Standard error of the mean.

${ }^{y}$ ANOVA analysis performed on log transformed data. Back-transformed data reported here.

${ }^{\mathrm{z}} \mathrm{CL}=$ Confidence limit. 
Concerning spore production, no statistical difference was observed among treatments, except the abaxial side of 1 st node leaves, which significantly produced more spores than the adaxial side of 5 th node leaves (Table 3 ).

Micropropagated plantlet bioassay. The species of PM pathogen used in the experiments did not have a significant effect on PM incidence and severity in either experimental run (Table 4). Both powdery mildew pathogens successfully infected micropropagated plantlets of $P$. glaberrima with disease incidence and severity values reaching 83 and 6\%, respectively, in plants infected with G. magnicellulatus, and 67 and $15 \%$, respectively, in those infected with Podosphaera sp. (Fig. 6).

\section{Discussion}

This study represents the first attempt to develop a set of laboratory tools to grow, maintain, and study PM pathogens of Phlox in vitro. The experiments carried out in this study identified the best conditions for two PM fungi (Podosphaera sp. and $G$. magnicellulatus) to grow in vitro on a detached leaf and a micropropagated plantlet bioassay and should facilitate future studies on host-microbe interactions, pathogen biology, and disease epidemiology. Additionally, our results could provide guidelines for working with other PM-ornamental systems as well.

The major limitation to Phlox production in nurseries and to its cultivation in landscapes is the plant's susceptibility to PM (Armitage 2008; Coombs 2017; Zale and Jourdan 2015). In the development of our detached leaf bioassay, we encountered several challenges as we aimed to maintain clean plants in the greenhouse as our source of plant material. Sulfur-based fungicides have been widely used as protectants against powdery mildews since 1000 BC (Nicot et al. 2002). Previous reports of in vitro PM bioassays in model systems indicated that sulfur could be washed off the leaves before use (Nicot et al. 2002; Peduto et al. 2013; Willocquet et al. 1996). However, the effect of sulfur on Phlox was unknown. In our studies, preliminary experiments showed that while vaporized sulfur
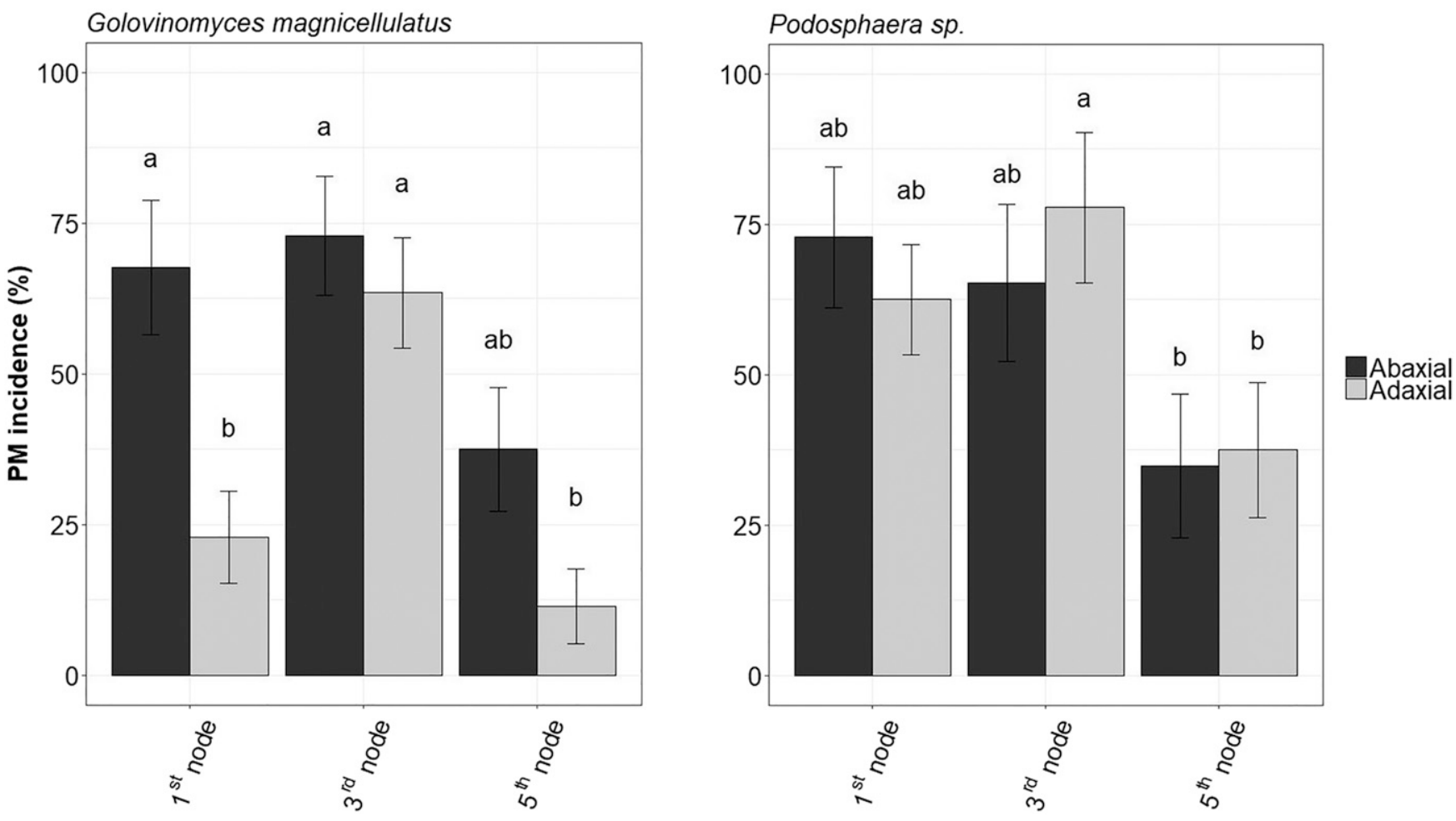

Fig. 5. Percentage of powdery mildew (PM) incidence developed on the abaxial and adaxial side of 1 st, 3rd, and 5th node leaves of Phlox paniculata inoculated with Golovinomyces magnicellulatus $(P=<0.0001)$ and Podosphaera sp. $(P=<0.0001)$. Columns represent the mean value of two runs of the experiment. Bars indicate $\pm S E$. Columns followed by the same letter are not statistically different according to Tukey's HSD test $(\alpha=0.05)$. Statistical analysis performed on arcsine-transformed data. Untransformed data are displayed.

Table 3. Effect of the phenology of the host tissue and side of the leaf surface on the size of the fungal colonies and the number of spores per unit of area produced by Golovinomyces magnicellulatus and Podosphaera sp. on detached Phlox paniculata leaves

\begin{tabular}{|c|c|c|c|c|c|c|c|c|c|}
\hline \multirow{3}{*}{$\frac{\text { Pathogen }}{\text { Golovinomyces magnicellulatus }}$} & \multirow{2}{*}{\multicolumn{2}{|c|}{ Parameter }} & \multicolumn{2}{|c|}{ 1st node } & \multicolumn{2}{|c|}{ 3rd node } & \multicolumn{2}{|c|}{ 5th node } & \multirow{3}{*}{$\frac{P \text { value }}{0.009}$} \\
\hline & & & \multirow{3}{*}{$\begin{array}{c}\text { Abaxial } \\
4.51 \mathrm{a}^{\mathrm{w}} \\
\pm 0.58\end{array}$} & \multirow{3}{*}{$\begin{array}{c}\text { Adaxial } \\
1.43 \mathrm{~b} \\
\pm 0.24\end{array}$} & \multirow{3}{*}{$\begin{array}{c}\text { Abaxial } \\
4.46 \mathrm{a} \\
\pm 0.4\end{array}$} & \multirow{3}{*}{$\begin{array}{r}\text { Adaxial } \\
\begin{array}{l}3.35 \mathrm{ab} \\
\pm 0.56\end{array}\end{array}$} & \multirow{3}{*}{$\begin{array}{c}\text { Abaxial } \\
\begin{array}{l}3.76 \mathrm{ab} \\
\pm 0.33\end{array}\end{array}$} & \multirow{3}{*}{$\begin{array}{c}\text { Adaxial } \\
4.36 \mathrm{ab} \\
\pm 0.46\end{array}$} & \\
\hline & Colony size $(\mathrm{mm})$ & Mean & & & & & & & \\
\hline & & $\mathrm{SE}^{\mathrm{x}}$ & & & & & & & \\
\hline & Spore/mm $\mathrm{mm}^{2}$ & Mean ${ }^{\mathrm{y}}$ & $169 \mathrm{ab}$ & $0.66 \mathrm{c}$ & $523.2 \mathrm{a}$ & $30.57 \mathrm{~b}$ & $399.4 \mathrm{ab}$ & $188.67 \mathrm{ab}$ & $<0.001$ \\
\hline & & $95 \% \mathrm{CL}^{\mathrm{z}}$ & $31.9,896.6$ & $0.29,1.5$ & $282.9,967.5$ & $5.19,180$ & $227.5,701.1$ & $69.9,508.9$ & \\
\hline \multirow[t]{4}{*}{ Podosphaera sp. } & Colony size $(\mathrm{mm})$ & Mean & $6.19 \mathrm{ab}$ & $4.13 \mathrm{bc}$ & $6.5 \mathrm{a}$ & $5.1 \mathrm{ab}$ & $4.6 \mathrm{abc}$ & $2.1 \mathrm{c}$ & $<0.001$ \\
\hline & & $\mathrm{SE}^{\mathrm{x}}$ & \pm 0.55 & \pm 0.76 & \pm 0.67 & \pm 0.91 & \pm 0.41 & \pm 0.43 & \\
\hline & Spore $/ \mathrm{mm}^{2}$ & Mean ${ }^{y}$ & $121.5 \mathrm{a}$ & $36.6 \mathrm{ab}$ & $66.7 \mathrm{ab}$ & $36.6 \mathrm{ab}$ & $73.7 \mathrm{ab}$ & $6.05 \mathrm{~b}$ & $<0.001$ \\
\hline & & $95 \% \mathrm{CL}^{\mathrm{z}}$ & $38.5,382.7$ & $14.2,94.2$ & $23.4,189.9$ & $15.1,88.5$ & $20.6,263.3$ & $1.5,24.2$ & \\
\hline
\end{tabular}

\footnotetext{
${ }^{w}$ Values with the same letter within each row are not significantly different according to Tukey HSD test $(\alpha=0.05)$.

$\times \mathrm{SE}=$ Standard error of the mean.

y ANOVA analysis performed on log transformed data. Back-transformed data reported here.

${ }^{\mathrm{z}} \mathrm{CL}=$ Confidence limit.
} 
applications were very efficient at protecting Phlox plants from PM infection in the greenhouse, they would not allow the pathogen to grow in vitro on leaves detached from those plants even after several water rinses and then undergoing disinfection (data not shown). In search for an alternative method to keep plants clean, we explored the use of a $20 \%$ raw milk solution, which we sprayed weekly on the entire plant surface. The mechanism by which milk sprays protect the plant from powdery mildew is poorly understood (Bettiol 1999; Kamel et al. 2017; Medeiros et al. 2012). While this study did not investigate such mechanism, the use of raw milk proved successful at controlling PM in the greenhouse and did not affect the ability of the pathogen to grow in vitro following disinfection of the leaves. Further investigations are needed to explore the mechanism by which raw milk protects Phlox from PM infections.

Inoculation techniques tested in this study encompassed the use of a hand-made tool made of an eyelash glued to a wooden stick; pressing an infected leaf onto a new disinfected leaf; and brushing inoculum using an artist paintbrush. The first method, described by Peries in 1962 (Nicot et al. 2002), allows for picking single conidia and depositing them in a precise location onto a clean surface, which facilitates generation of single-spore colonies. The technique was described as being more successful than the use of fine needles, due to greater suppleness of the eyelash and possibly to higher static electricity, and was used successfully in this study to generate pure cultures of the two PM isolates. Pressing a leaf segment bearing one PM culture up to eight different places on a clean leaf did not result in different colony incidence and allowed quick and easy generation of multiple colonies from little inoculum. Finally, an artist paintbrush, described numerous times in the literature (Kenyon et al. 1995; Leus et al. 2006; Nicot et al. 2002), was used to successfully and uniformly inoculate entire micropropagated plantlets. To limit the loss of inoculum, we thinned the number of bristles on each paintbrush and observed under a dissecting microscope that less spores were caught up after plantlet inoculation (data not shown).

When using a leaf-based bioassay, the longer the leaves are maintained in good conditions, the less frequent the transfer of PM onto new healthy leaves is needed. Benzimidazole has been used in numerous leaf bioassay studies to protect cultures from contaminations, and to delay tissue senescence (Arabi and Jawhar 2002; Azmat et al. 2013; Linde and Debener 2003; Nicot et al. 2002; Surlan-Momirovic et al. 2016). Use of tetracycline has been reported by Bardin et al. (1999) to reduce occurrence of contaminants as well. However, when comparing different media for their ability to maintain leaf quality over time, in this study we did not observe a significant effect of benzimidazole nor tetracycline in the medium when used individually compared with nonamended medium. However, when used in combination, leaf damage was significantly higher compared with nonamended medium.

To define the best conditions for PM growth in a detached leaf bioassay, we considered the effect of different factors: age of the culture used to inoculate leaves, phenology of the host tissue, and side of the leaf surface onto which inoculum is placed. Regardless of the PM fungus tested (G. magnicellulatus or Podosphaera sp.), the use of 18-day-old cultures resulted in the generation of a higher number of colonies that were larger and on which more spores were produced, compared with the use of 14- or 22-day-old cultures. PM conidia are produced in chains on the mycelium. Nicot et al. (2002) described three stages of conidial formation. Among these, mature conidia, which are connected to the chain by a papillum on the rounded end, are only observed in the last stage. In his studies on PM of lettuce, Schnathorst (1959) showed that in G. chicoracearum only the last spore (oldest) is able to infect the host, even if all spores in the chain are able to germinate, suggesting that age of the spores is an important factor in conidial infection. These observations are in accordance with the results of this study in which younger cultures (14-day-old) did not infect the host as much as 18-day-old cultures. Furthermore, Nicot et al. (2002) mentioned that conidial production ceases with senescence of the host. In our media experiment, a high percentage of leaf necrosis and chlorosis were observed on detached leaves 20 days post plating (data not shown). It is likely that as leaf health degraded, inoculum also started to senesce. On the basis of these observations, 18-day-old cultures were subsequently used to test the additional experimental factors.

$P$. paniculata $\mathrm{cv}$. Starfire shows anthocyanin pigment on young leaves, which then disappears as the leaves mature. This factor, as well as the side of the leaf on which the pathogen spores land, might influence PM growth. Ontogenic or age-related resistance of the host has been reported in different PM systems, for example strawberry (Asalf et al. 2014; Sombardier et al. 2009) and grape (Ficke et al. 2002; Gadoury et al. 2003). In strawberries, Asalf et al. (2014) have found that relative disease severity declined with increasing leaf age. Moreover, Sombardier et al. (2009) reported a 10 times higher infection efficiency on the abaxial side of strawberry leaves, compared with the adaxial side. In the grape PM system, host susceptibility has been found to be centered around bloom (Gadoury and Seem 1995; Gadoury et al. 1997, 2000), with 4-week-old berries being nearly immune to the disease (Ficke et al. 2002). Ficke et al. (2002) also suggest that resistance in grape berries is unlikely linked to cuticle or cell wall thickness. In Phlox, Jarosz et al. (1982) did not find a correlation between leaf cuticle thickness and resistance to powdery mildew when comparing different Phlox species with related differences in cuticle thickness, suggesting that it is unlikely that a difference in cuticle thickness between the adaxial and abaxial side of the leaf plays a role in appressorium penetration. Even though PM incidence on the abaxial side of the leaf was higher in some of our experiments, after 2 weeks of culturing, we observed that the leaf tended to curl up, which makes accessibility to the inoculum and consequent measuring or rating very difficult. Moreover, the side of the leaf surface on which inoculum was placed had an inconsistent

Table 4. Summary statistics of the effect of the powdery mildew (PM) species on the incidence and severity of powdery mildew infection observed on micropropagated Phlox glaberrima plantlets

\begin{tabular}{ccccccc}
\hline & & \multicolumn{2}{c}{ PM incidencey } & & \multicolumn{2}{c}{ PM severity } \\
Factor tested & Experimental run & $\boldsymbol{F}$ & $\boldsymbol{P}$ value & & $\boldsymbol{F}$ & $\boldsymbol{P}$ value \\
\hline PM species $^{\mathrm{z}}$ & 1 & 0.38 & 0.55 & & 0.96 & 0.35 \\
& 2 & 0.38 & 0.55 & & 1.62 & 0.23 \\
\hline
\end{tabular}

y ANOVA analysis performed on arcsine-transformed data.

z Two PM species were tested: Golovinomyces magnicellulatus and Podosphaera $\mathrm{sp}$.

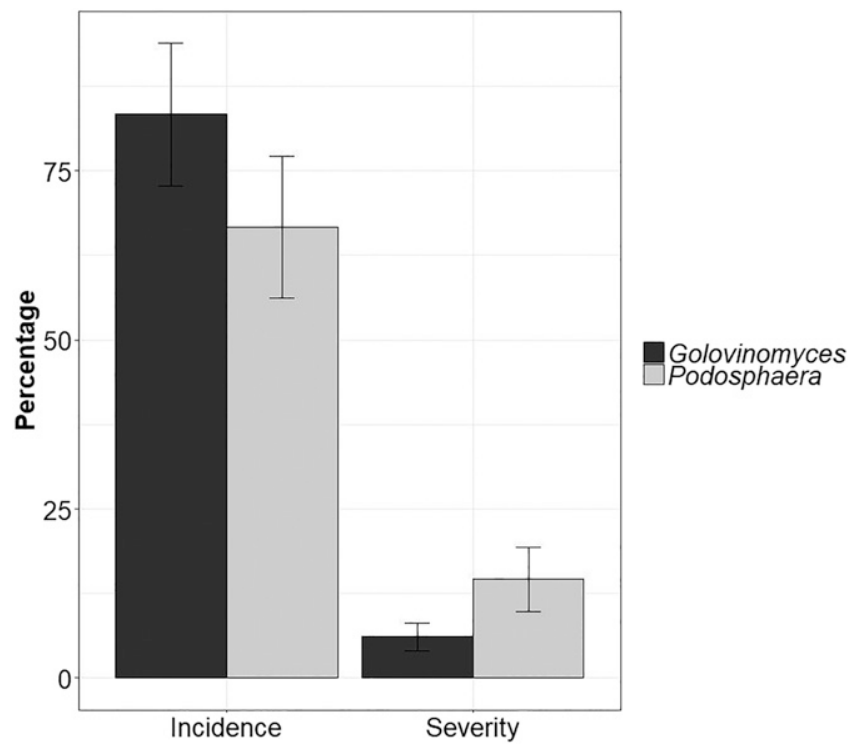

Fig. 6. Incidence $(P=0.37)$ and severity $(P=0.115)$ of powdery mildew $(\mathrm{PM})$ recorded on micropropagated plantlets of Phlox glaberrima inoculated with Golovinomyces magnicellulatus and Podosphaera sp. Columns represent the mean value of two runs of the experiment. Bars indicate \pm SE. Statistical analysis performed on arcsine-transformed data. Untransformed data are displayed. 
effect across the three experimental runs. Consequently, depending on the test's purpose and type of data assessed, it might be more practical to use the adaxial side of the leaf for further tests on PM of Phlox. When using the adaxial side of the leaf, 3rd node leaves would significantly sustain more powdery mildew growth than 5 th node leaves, indicating a possible ontogenic resistance mechanism in Phlox. Further investigations are needed to explore the difference in PM infection between the two sides of the leaf.

In this study, we also explored the use of micropropagated Phlox plantlets to carry out in vitro PM studies. Based on our results, the use of micropropagated plantlets could provide several benefits. First, micropropagated plantlets are more likely to be PM-free since grown in vitro, which would result in the avoidance of laborious fungicide sprays and possible contaminations. Second, Phlox is a broad genus that encompasses species with very diverse leaf phenology. The use of plantlets over detached leaves would allow standardizing the bioassay when using different Phlox species. Lastly, using plantlets over detached leaves allows evaluating plant response from an entire plant, compared with a response from an injured, detached leaf. However, micropropagated plantlets are slow to grow (approximately 5 months), and propagation is time-consuming. Moreover, the plants are grown in microenvironments that could affect the leaf tissue and the interaction with the pathogen.

In our experiments we successfully infected micropropagated $P$. glaberrima plantlets. While both inocula resulted in high disease incidence, low severity was observed. This could be due to the length of time left for the pathogens to develop ( 3 weeks) and the amount of inoculum used to inoculate the plantlets (one 9-mmdiameter colony per plantlet). This method should nevertheless prove easier for further studies on interaction between the host and its pathogen.

In conclusion, in this study we successfully developed two laboratory bioassays and optimized the experimental conditions to use these tools to study PM pathogens of Phlox in vitro. Further studies focused on screening perennial Phlox germplasm for susceptibility to PM through these bioassays are underway and should provide additional information on the practical applications of these tools. Moreover, the bioassays developed here could serve as a model for other PM-ornamental crop systems.

\section{Acknowledgments}

The research described in this paper represents a portion of the dissertation submitted by C. Farinas to the Office of Graduate Studies of The Ohio State University to partially fulfill requirements for the Ph.D. degree in plant pathology. We thank M. Kelly, J. Morse, M. Bellizzi, S. Lin, I. Emmanuel, and M. Moodispaw for technical assistance, as well as C. Tubesing at Holden Arboretum (OH) and L. Matthew at Lewis Ginter Botanical Garden (VA) for providing the PM isolates used in this study.

\section{Literature Cited}

Arabi, M. I. E., and Jawhar, M. 2002. The ability of barley powdery mildew to grow in vitro. J. Phytopathol. 150:305-307.

Armitage, A. M. 2008. Herbaceous Perennial Plants. A Treatise on Their Identification, Culture, and Garden Attributes, 3rd ed. Stipes Pub. LLC, Champaign, IL. Pages 787-800.

Asalf, B., Gadoury, D. M., Tronsmo, A. M., Seem, R. C., Dobson, A., Peres, N. A., and Stensvand, A. 2014. Ontogenic resistance of leaves and fruit, and how leaf folding influences the distribution of powdery mildew on strawberry plants colonized by Podosphaera aphanis. Phytopathology 104:954-963.

Azmat, M. A., Khan, A. A., Cheema, H. M. N., Ashraf, M., and Niaz, S. 2013. Detached leaf assay coupled with microscopic conidial quantification: An efficient screening method for powdery mildew resistance in pea. Int. J. Agric. Biol. 15:957-962.

Bardin, M., Carlier, J., and Nicot, P. C. 1999. Genetic differentiation in the French population of Erysiphe cichoracearum, a causal agent of powdery mildew of cucurbits. Plant Pathol. 48:531-540.

Bettiol, W. 1999. Effectiveness of cow's milk against zucchini squash powdery mildew (Sphaerotheca fuliginea) in greenhouse conditions. Crop Prot. 18:489-492.

Bir, R. E., and Conner, J. L. 2002. Mildew resistant garden Phlox. N.C. State University. Poster Session Paper, Proceedings of the International Plant Propagator's Society.

Braun, U., Cook, R. T. A., Inman, A. J., and Shin, H.-D. 2002. The taxonomy of the powdery mildew fungi. Pages 13-55 in: The Powdery Mildews: A
Comprehensive Treatise. R. R. Langer, W. R. Bushnell, A. J. Dik, and T. L. W. Carver, eds. American Phytopathological Society, St Paul, MN.

Coombs, G. 2017. Phlox for the mid-Atlantic region. Research Report. Mt. Cuba Center.

De Mendiburu, F. 2017. agricolae: Statistical Procedures for Agricultural Research. R package version 1.2-8. https://CRAN.R-project.org/package= agricolae

Farinas, C., and Peduto Hand, F. 2017. Genotypic characterization of isolates of Golovinomyces magnicellulatus, the biotrophic powdery mildew pathogen of Phlox. (Abstr.) Phytopathology 107(12S):S5.122.

Ficke, A., Gadoury, D. M., and Seem, R. C. 2002. Ontogenic resistance and plant disease management: a case study of grape powdery mildew. Phytopathology 92:671-675.

Gadoury, D. M., and Seem, R. C. 1995. Development of ontogenic resistance to powdery mildew (Uncinula necator) in fruit of Concord grapevines. (Abstr.) Phytopathology 85:1149.

Gadoury, D. M., Seem, R. C., Ficke, A., and Wilcox, W. F. 2003. Ontogenic resistance to powdery mildew in grape berries. Phytopathology 93:547-555.

Gadoury, D. M., Seem, R. C., and Wilcox, W. F. 1997. Early ontogenic resistance to powdery mildew in Chardonnay and Riesling grapes. (Abstr.). Phytopathology 87 (suppl.):S31.

Gadoury, D. M., Seem, R. C., and Wilcox, W. F. 2000. The early development of ontogenic resistance to powdery mildew in fruit of Vitis labruscana and Vitis vinifera grapevines. Proc. Int. Workshop Grapevine Downy and Powdery Mildew, 3rd. SARDI Res. Rep. Ser. 50:111-112.

Hawke, R. 1999. Plant evaluation notes. An evaluation report of selected Phlox species and hybrids. Chicago Botanic Garden. Issue 13.

Hawke, R. 2011. Plant evaluation notes. A comparative study of Phlox paniculata cultivars. Chicago Botanic Garden. Issue 35.

Hermann, F. 1994. Pages 198-199 in: Phlox, Stauden-und Polsterphlox. Eugen Ulmer GmbH \& Co, Stuttgart, Germany.

Jarosz, A. M., Sheets, M., and Levy, M. 1982. Cuticle thickness in Phlox and resistance to powdery mildew: An unreliable line of defense. Am. J. Bot. 69: 824-828.

Kamel, S. M., Ketta, H. A., and Emeran, A. A. 2017. Efficacy of raw milk and whey against cucumber powdery mildew disease caused by Sphaerotheca fuliginea (Schlecht.) Pollacci under plastic house conditions. Eg. J. Bio. Pest Con. 27:135-142.

Kenyon, D. M., Dixon, G. R., and Helfer, S. 1995. Culture in vitro of Phododendron and Erysiphe sp. Plant Pathol. 44:350-354.

Leus, L., Dewitte, A., Huylenbroeck, J. V., Vanhoutte, N., Bockstaele, E. V., and Hofte, M. 2006. Podosphaera pannosa (syn. Sphaerotheca pannosa) on Rosa and Prunus spp.: Characterization of pathotypes by differential plant reactions and ITS sequences. J. Phytopathol. 154:23-28.

Linde, M., and Debener, T. 2003. Isolation and identification of eight races of powdery mildew of roses (Podosphaera pannosa) (Wallr.: Fr.) de Bary and the genetic analysis of the resistance gene Rpp1. Theor. Appl. Genet. 107: 256-262.

Locklear, J. H. 2011. Phlox. A Natural History and Gardener's Guide. Timber Press, Inc.

Madden, L. V., Hughes, G., and van den Bosch, F. 2007. The study of plant disease epidemics. The American Phytopathological Society, St. Paul, MN.

Matsuda, S., and Takamatsu, S. 2003. Evolution of host-parasite relationships of Golovinomyces (Ascomycete: Erysiphaceae) inferred from nuclear rDNA sequences. Mol. Phylogenet. Evol. 27:314-327.

McGrath, M. T., and Shishkoff, N. 2001. Resistance to triadimefon and benomyl: Dynamics and impact on managing cucurbit powdery mildew. Plant Dis. 85: 147-154.

Medeiros, F. H. V., Bettiol, W., Souza, R. M., Alves, E., Pinto, Z. V., and Lost, R. 2012. Microorganisms, application timing and fractions as players of the milkmediated powdery mildew management. Crop Prot. 40:8-15.

NASS. 2014. https://quickstats.nass.usda.gov/\#CD852985-E809-3D46-88F137DA2C7134B8

Nicot, P. C., Bardin, M., and Dik, A. J. 2002. Basic methods for epidemiological studies of powdery mildews: Culture and preservation of isolates, production and delivery of inoculum, and disease assessment. Pages 83-99 in: The Powdery Mildews: A Comprehensive Treatise. R. R. Langer, W. R. Bushnell, A. J. Dik, and T. L. W. Carver, eds. American Phytopathological Society, St Paul, MN.

Peduto, F., Backup, P., Hand, E. K., Janousek, C. N., and Gubler, W. D. 2013 Effect of high temperature and exposure time on Erysiphe necator growth and reproduction: revisions to the UC Davis powdery mildew risk index. Plant Dis. 97:1438-1447.

Pinheiro, J., Bates, D., DebRoy, S., Sarkar, D., and R Core Team. 2017. nlme: Linear and Nonlinear Mixed Effects Models. R package version 3.1-131. https://CRAN.R-project.org/package=nlme

Schnathorst, W. C. 1959. Spread and life cycle of the lettuce powdery mildew fungus. Phytopathology 49:464-468.

Sombardier, A., Savary, S., Blancard, D., Jolivet, J., and Willocquet, L. 2009 Effects of leaf surface and temperature on monocyclic processes in Podosphaera aphanis, causing powdery mildew of strawberry. Can. J. Plant Pathol. 31:439-448. 
Surlan-Momirovic, G., Flath, K., Silvar, C., Brankovic, G., Kopahnke, D., Knezevic, D., Schliephake, E., Ordon, F., and Perovic, D. 2016. Exploring the Serbian GenBank barley (Hordeum vulgare L. subsp. vulgare) collection for powdery mildew resistance. Gener. Resour. Crop Evol. 63:275-287.

Thompson, H. S., and Svejda, F. J. 1965. Resistance to powdery mildew in perennial Phlox. Can. J. Plant Sci. 45:258-263.

Walsh, P. S., Metzger, D. A., and Higuchi, R. 1991. Chelex 100 as a medium for simple extraction of DNA for PCR-based typing from forensic material. BioTechniques 10:506-513.
Willocquet, L., Colombet, D., Rougier, M., Fargues, J., and Clerjeau, M. 1996. Effects of radiation, especially ultraviolet $\mathrm{B}$, on conidial germination and mycelial growth of grape powdery mildew. Eur. J. Plant Pathol. 102:441-449.

Zale, P., and Jourdan, P. 2015. Genome size and ploidy and Phlox paniculata and related germplasm in subsections Paniculatae and Phlox. J. Am. Soc. Hortic. Sci. 140:436-448

Zale, P., Robarts, D. H. W., and Jourdan, P. 2016. Genome size and ploidy levels of creeping phlox and related germplasm of mat-forming taxa from eastern and western North America. Sci. Hortic. 203:53-61. 